\title{
Non-uniform illumination correction in infrared images based on a modified fuzzy c-means algorithm
}

\author{
Marios Dimitrios Vlachos, Evangelos Spyros Dermatas \\ University of Patras, Patras, Greece
}

Correspondence: Marios Dimitrios Vlachos. Address: Department of Electrical and Computer Engineering, University of Patras, Kato Kastritsi, 26500, Rio Patras, Greece. Telephone: 30-261-099-6476. Email: mvlachos@teemail.gr.

\section{Abstract}

The correction of non-uniform illumination and the elimination of shading artifacts is an important preprocessing task used in a great number of image processing applications such as segmentation, registration or quantitative analysis. Although, a careful and accurate set up of the image acquisition system may degrade the importance of a brightness normalization algorithm, non-uniform illumination appears due to the interaction of objects and light on the scene requires retrospective shading correction. The image formation process and the corresponding shading effects are described by a linear image formation model, which consists of a multiplicative and an additive shading component.

In this paper a novel brightness normalization method is proposed to eliminate the non-uniform illumination effects. The method is based on the application of a fuzzy c-means algorithm (FCM) only on the background part of the acquired image, where the objective function is modified to take into account local information of each pixel in the estimation of the multiplicative and the additive shading components. The modified FCM algorithm is iterative, as the standard FCM, and at each iteration the multiplicative and the additive shading components are re-estimated based on the cluster centers and the membership of each pixel in a specific cluster. Brightness correction is performed by the inverse of the image formation model after FCM convergence.

Experiments were conducted in a database of both real and artificial infrared images. The experimental results show that the proposed method decreases significantly the non-uniform illumination effects and does not introduce brightness variations if the background is uniform.

\section{Key words}

Infrared images, Shading, Non-uniform illumination, Modified fuzzy c-means, Image formation model

\section{Introduction}

The problem of shading and of presence of intensity inhomogeneities is common in many image modalities. Although, this problem has minor impact on visual image interpretation due to the fact that human perception has the ability to deduce the real image content from a distorted image its impact in automatic image processing or analysis cannot be assumed 
negligible. Thus, the correction of shading and the compensation of non-uniform illumination is an important preprocessing step in many image processing tasks.

In general, shading can be either object-independent or object-dependent. In the first case, an imperfect set up of the image acquisition system introduces the shading and may be corrected by calibration methods, while in the latter shading produced from the interaction between the objects in the scene and can be corrected only retrospectively by using information from the acquired images.

In case of infrared images shading is object-dependent and thus removing the non-uniform illumination effects can be achieved retrospectively. A retrospective shading correction method must correct the shading only when it is present and to not distort the shading-free images.

The formation of an image $\mathrm{Y}$ is an interaction between objects and illumination on the scene. The relation between the acquired image and the shading-free image $\mathrm{X}$ can be described by the following linear model:

$$
Y=A \cdot X+B
$$

where $\mathrm{A}$ and $\mathrm{B}$ are the multiplicative and additive shading component respectively. The objective of the shading correction is to derive the shading-free image $\mathrm{X}$ from the acquired image $\mathrm{Y}$ by estimating the multiplicative $\mathrm{A}$ and additive $\mathrm{B}$ shading components. This is done by inverting the linear model as follows:

$$
X=\frac{Y-\hat{B}}{\hat{A}}
$$

where $\hat{A}$ and $\hat{B}$ are the estimations of the multiplicative and the additive shading component respectively.

Many approaches simplify the previous problem by assuming that only one component (either the multiplicative A or the additive B) has distorted the shading-free image X. In these cases shading correction is achieved by using one from the above equations depending on the assumed model:

$$
\begin{gathered}
X=Y-\hat{B}+C \\
X=\frac{Y}{\hat{A}} \cdot C
\end{gathered}
$$

where $\mathrm{C}$ is a constant that used to normalize the image intensities to lie in a desired range. For object-independent shading correction, the multiplicative A or the additive B shading component, may be estimated by acquiring one calibrating image. On the other hand, for object-dependent shading correction the solution calls for a retrospective shading method (shading components should be estimated from the acquired image $\mathrm{Y}$ ).

A lot of retrospective shading correction methods have already been presented in the literature ${ }^{[1-5]}$. For instance, linear filtering ${ }^{[1,2]}$ assumes that only the additive shading component has distorted the image and that it can be estimated by filtering the acquired image with a low pass filter. The estimation of the shading-free image is then performed using equation (3). A similar approach, homomorphic filtering ${ }^{[2,4]}$, assumes that only the multiplicative shading component is present in the acquired image and its estimation performed by low pass filtering of the acquired image in the log domain. The shading-free image obtained using equation (4). In both approaches the success in the estimation of the shading components depends on the selection of an appropriate low pass filter. 
Morphological filtering ${ }^{[5]}$ method based mainly on two assumptions. First of all, it assumes that the background has darker or brighter intensity values than the other objects of the scene and that the size of the object of interest is smaller than the background variations. Thus, the selection of an appropriate morphological operator can be used for the estimation of the background which represents either the multiplicative or the additive shading component. In such case equation (3) or (4) can be used for estimation of the shading-free image.

A different approach ${ }^{[1,3]}$ assumes that the intensity variations of the background or of the object class can be estimated by fitting a shading model to the intensity values of pre-selected labels of each class. Usually, a second order polynomial is used as the function of the model for least-squares fitting. This function may represent either the multiplicative or the additive shading component and equations (3), (4) can be used to estimate the shading-free image. Moreover, two functions can be used to estimate both intensity variations of the background and object class and in the sequel the multiplicative and the additive shading components may be derived from these estimations. The most important issue in this method is the selection of the labels of each class which can be done manually or automatically.

A recent approach ${ }^{[3]}$, assumes that the image formation model (equation (1)) increases the degree of uncertainty in the image. A representative measure of uncertainty is the entropy. Second order polynomials are used to model the multiplicative and the additive shading components and then their parameters are varying until the entropy $\mathrm{H}$ of the image $\mathrm{X}$ is minimized.

A YUV shading correction technique applicable to colour images is proposed ${ }^{[6]}$. This technique involves, the conversion of the acquired RGB image to its YUV representation, the application of the linear filtering approach only to the intensity channel $\mathrm{Y}$ (letting the chrominance channels $\mathrm{U}$ and $\mathrm{V}$ unchanged) and the back conversion of the processed image to its RGB representation. Experiments conducted on images with synthesized shading show feasibility of the approach.

A method to normalize the luminosity and the contrast in retinal images, both intra- and inter- image is presented ${ }^{[7]}$. Due to the acquisition process, very often these images are non-uniformly illuminated and exhibit local luminosity and contrast variability and thus the automatic analysis for diagnostic purposes becomes very difficult. This method is based on the estimation of luminosity and contrast variability in the background part of the image and the subsequent compensation of this variability in the whole image. Moreover, it improves automatic fundus analysis and helps eye specialists in their visual examination of retinal images.

A method to correct non-uniform illumination in transmission electron microscopy (TEM) is proposed ${ }^{[8]}$. TEM images suffer from variations in brightness due to electron imaging defects or non-uniform support films and specimen staining. These variations render image processing operations such as segmentation more difficult. The correction requires estimation of the global illumination field which is performed using only image intensity gradients. This method has a solution in closed-form which computed very fast.

An additive retrospective non-parametric algorithm for the correction of the inhomogeneous intensity background of images, commonly known as shading, is presented ${ }^{[9]}$. The algorithm assumed that an original unbiased image was corrupted by slowly-varying shading that could be estimated from the signal envelope in a process analogous to amplitude modulation detection. Unlike other filtering algorithms, the algorithm did not require pre-processing, parameter setting, user interaction, computationally intensive optimisation algorithms or a restriction in size of the objects of interest relative to the scale of background variations. The algorithm provided satisfactory results for artificial and microscopical images.

The interaction between objects in real space, the illumination, and the camera frequently leads to a situation in which a microscope image exhibits significant shading across the field of view. In general this shading effect is undesirable and requires elimination, especially for quantitative microscopy. Authors starting with a simple mathematical model, develop procedures to correct images for the shading thus introduced in the image-formation process ${ }^{[10]}$. Two cases are 
distinguished: the a priori, which assumes the availability of calibration images in addition to the images of interest; and the a posteriori, which assumes that only the recorded images of interest are available.

A fully automatic blind retrospective shading correction method based mainly on a minimization of a multi-objective criterion is presented ${ }^{[11]}$. The proposed method assumes that the acquired image has distorted from a multiplicative and an additive shading component and thus can be adequately described by the linear image formation model. The estimation of the shading-free image is based on parametric estimation of the multiplicative and the additive shading component and the consequent application of the inverse image formation model. First of all, an initial estimation of the shading-free image is performed by the minimization of the multi-objective function of an appropriate image criterion. Secondly, the multiplicative and the additive shading components are estimated, based on assumptions about their frequency content and then, they median filtered. Finally, an estimation of a shading-free image is obtained using the above estimations for the components and the application of the inverse image formation model.

A FCM based algorithm that simultaneously estimates the shading effect while segmenting the image is presented ${ }^{[12]}$. A multiplier field term that models the intensity variation is incorporated into the FCM objective function which is minimized iteratively. The bias field is estimated based on the current tissue class centroids and the membership values of the voxels and then smoothed by an iterative low-pass filter. The algorithm is evaluated on clinical breast MR images.

A novel algorithm for fuzzy segmentation of magnetic resonance imaging (MRI) data and estimation of intensity inhomogeneites using fuzzy logic is proposed ${ }^{[13,14]}$. This algorithm is formulated by modifying the objective function of the standard FCM algorithm to compensate for such inhomogeneites and to allow the labeling of a pixel to be influenced by the labels in its immediate neighborhood. Experimental results are conducted on both synthetic images and MR data and demonstrate the effectiveness and efficiency of the proposed algorithm.

A novel brightness normalization method is used to eliminate the non-uniform illumination effects in infrared images ${ }^{[15]}$. The algorithm is based on a FCM algorithm, where the objective function is modified to take into account local information of each pixel related both on multiplicative and additive shading effects. At each iteration, the modified FCM algorithm re-estimates the cluster centroids and the membership weights by processing the adopted shading features. Brightness correction is performed by the inverse of the image formation model after FCM convergence.

In this paper, a brightness normalization method is proposed to eliminate the non-uniform illumination effects in infrared images ${ }^{[13-15]}$. The algorithm is based on the application of a FCM algorithm only on the background part of the acquired image, where the objective function is modified to take into account local information of each pixel in the estimation of the multiplicative and the additive shading components. The modified FCM algorithm is iterative, as the standard FCM, and at each iteration the multiplicative and the additive shading components are re-estimated based on the cluster centers and the membership of each pixel in a specific cluster. Brightness correction is performed by the inverse of the image formation model (equation (2)) after FCM convergence.

The main improvements of the proposed method comparing with the methods presented ${ }^{[13-15]}$ are that it uses only the background part of the image in order to estimate the multiplicative and the additive shading components while the method presented ${ }^{[15]}$ uses the whole image without preprocessing. That has the advantage that the modified FCM algorithm focuses only in the part of the image which suffers from non-uniform illumination and inhomogeneities. Moreover, both the multiplicative and the additive shading components are adopted in the proposed method, while only an additive component used ${ }^{[13,14]}$. By using the full image formation model the proposed method achieves a more accurate removal of shading.

\section{Proposed algorithm}

The proposed method is summarized in the flowchart shown in Figure 1.

Published by Sciedu Press 


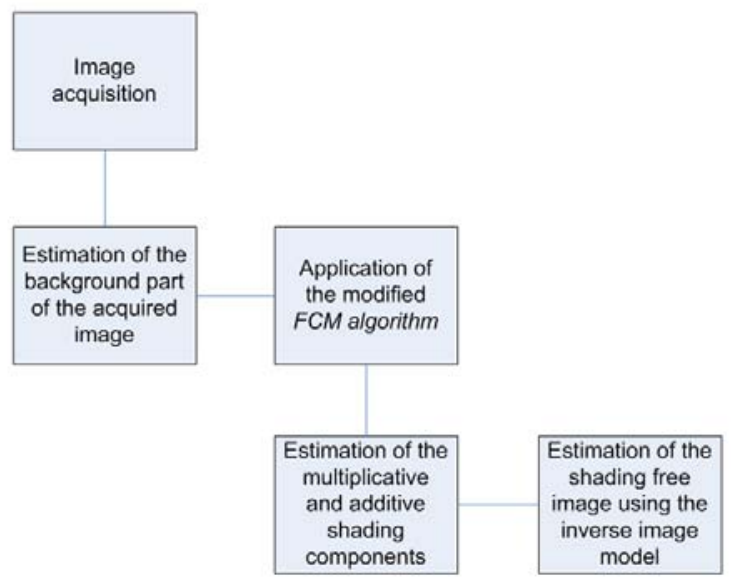

Figure 1. Flowchart of the proposed algorithm

Image acquisition. The original infrared image acquired under infrared light using an inexpensive CCD camera. The finger is placed between the camera and the light source which consists of a row of infrared leds (five elements) with adjustable illumination. Although, a careful image acquisition setup can be achieved by selecting uniform light sources situated in appropriate distances between them the acquired infrared images will suffer from shading and non-uniform illumination mainly due to the physiology of the tissue in finger region. Thus, a shading correction algorithm must be applied to obtain the shading-free image which can make automatic segmentation or registration algorithms applicable.

Estimation of the background. The process of infrared image acquisition can be described by the following model:

$$
Y=f(X)=f\left(X_{b}+X_{f}\right)
$$

where $\mathrm{X}$ is the original shading-free image, $\mathrm{X}_{\mathrm{b}}$ is the background part of the shading-free image, $\mathrm{X}_{\mathrm{f}}$ is the foreground part of the shading-free image and function $\mathrm{f}($.) represents the acquisition transformation.

In infrared images, the background part of the image $X_{b}$ is the part of image free of any vascular structure. Vascular structures belong to the foreground part of the image $X_{\mathrm{f}}$ and they are modelled as an additive term to the background part of the image.

Although assumptions for the foreground part of the image cannot be easily made, the background part of the image can be statistically modelled as:

$$
X_{b} \sim N\left(\mu_{b}, \sigma_{b}\right)
$$

where $\mu_{\mathrm{b}}$ is the mean value of the background part of the image, $\sigma_{\mathrm{b}}$ is the standard deviation of the background part of the image and $\mathrm{N}(.,$.$) is a white random field with mean value representing the uniform brightness value and standard deviation$ representing the variability in brightness due to the interaction between objects in the scene. The above model can be simplified by assuming that $\mu_{\mathrm{b}}=0$ and $\sigma_{\mathrm{b}}=1$. This assumption is reasonable as any additive or multiplicative factor can be involved in the acquisition function $\mathrm{f}$.

The relation between the acquired infrared image of finger and the shading-free image which expressed by the acquisition transformation function $\mathrm{f}($.) can be described by the linear model (1). Y represents the acquired infrared image, $\mathrm{X}$ the shading-free image and A, B the multiplicative and additive shading component respectively. Both multiplicative and additive shading components are space-dependent scalar functions and can therefore be considered as images themselves. 
The original shading-free image is estimated after the estimation of the multiplicative and additive shading and applying the inverse model (equation (2)). Both A and B are assumed to be spatially smooth, which means that illumination irregularities do not present rapid changes.

The estimation of the multiplicative and the additive shading components can be achieved by considering their effect only on the background part of the image. Combining equations (1) and (5) it yields:

$$
\begin{aligned}
& Y=f(X)=A \cdot X+B \Rightarrow \\
& Y=A \cdot\left(X_{b}+X_{f}\right)+B \Rightarrow \\
& Y=A \cdot X_{b}+A \cdot X_{f}+B
\end{aligned}
$$

If the analysis is restricted only to the background part of the image:

$$
Y=A \cdot X_{b}+B, \forall(x, y) \in \text { Background }
$$

since by definition $\mathrm{X}_{\mathrm{f}}=0$ in background.

Using the statistical model of the background part of the image $\mathrm{X}_{\mathrm{b}}$ the statistical description of the background pixels is:

$$
Y=N(A, B), \forall(x, y) \in \text { Background }
$$

The estimation of the multiplicative and additive shading components requires the extraction of the set of pixels which belong with high confidence to the background. To achieve this, the following assumptions are made for any pixel belonging to a window neighborhood of size $\mathrm{N} \times \mathrm{N}$ : (1) the multiplicative and additive shading components are constant, (2) background pixels cover at least the $50 \%$ of the neighborhood and (3) the brightness values of the background pixels are different from those of the foreground pixels. The above assumptions are valid for the infrared images. The first is based on the hypothesis of the model that the multiplicative and additive shading components are spatially smooth. The second is valid in the majority of image regions and the third allows discriminating background from foreground pixels based only on their brightness value.

For each pixel $(\mathrm{x}, \mathrm{y})$ of the image the mean value and the standard deviation inside a $\mathrm{NxN}$ window neighborhood are estimated. As estimator for the mean value $\mu_{\mathrm{b}}$ the sample mean $\hat{\mu}_{\mathrm{b}}$ is used and as estimator for the standard deviation $\sigma_{\mathrm{b}}$ the sample standard deviation $\hat{\sigma}_{b}$. Pixel $(\mathrm{x}, \mathrm{y})$ is considered to belong to the background if its brightness is near to the mean brightness value estimated inside the window neighborhood of size $\mathrm{N} \times \mathrm{N}$ or equivalently the following distance $\mathrm{d}$ is lower than a predefined threshold:

$$
d=\left|\frac{Y(x, y)-\hat{\mu}_{b}}{\hat{\sigma}_{b}}\right| \leq \text { threshold }
$$

This is mathematically expressed by saying that pixel (x,y) belongs to background if its Mahalanobis distance from $\hat{\mu}_{b}$ is lower than a given threshold $t$. As regards threshold $t$, its value was set to 1 , which means that, with normally distributed luminosity, about $68 \%$ of the window pixels are retained as background (note that Mahalanobis distance is by definition normalized for each pixel by its standard deviation).

After the extraction of the background pixels using equation (10), the modified FCM algorithm is applied and the multiplicative and additive shading components are estimated as follows. 
Application of the modified FCM algorithm on the background part of the image. The objective function of the standard FCM algorithm for separating the data $\mathrm{x}$ into $\mathrm{c}$ clusters is given by the following equation:

$$
J=\sum_{i=1}^{c} \sum_{k=1}^{N} u_{i k}^{p} \cdot\left\|x_{k}-v_{i}\right\|^{2}
$$

where $\mathrm{x}_{\mathrm{k}}$ are the pixels belonging only to the background part of the image $\mathrm{X}_{\mathrm{b}},\left\{v_{i}\right\}_{i=1}^{c}$ are the centroids of the clusters and the array $\left[u_{i k}\right]=U$ represents a partition matrix, $U \in U$, namely

$$
u\left\{u_{i k} \in[0,1] \mid \sum_{i=1}^{c} u_{i k}=1 \forall k \text { and } 0<\sum_{k=1}^{N} u_{i k}<N \forall i\right\}
$$

The parameter $p$, called fuzzifier, is a weighting exponent on each fuzzy membership and determines the amount of fuzziness of the resulting classification. This function is minimized when high membership values are assigned to pixels belonging to the same class.

The objective function of the standard FCM is modified by adding a term that allows the labelling of each pixel to be influenced by the labels in its immediate neighbourhood ${ }^{[10,11]}$. The addition of this term biases the solution toward piecewise-homogenous labelling. It exploits the fact that pixels belonging to the same neighbourhood are spatially correlated and it is common to belong in the same class. The modified objective function is given by the following formula:

$$
J_{a b}=\sum_{i=1}^{c} \sum_{k=1}^{N} u_{i k}^{p} \cdot\left\|x_{k}-v_{i}\right\|^{2}+\frac{n_{R}}{N_{R}} \cdot \sum_{i=1}^{c} \sum_{k=1}^{N} u_{i k}^{p} \cdot\left(\sum_{x_{r} \in N_{k}}\left\|x_{r}-v_{i}\right\|^{2}\right)
$$

where $\mathrm{N}_{\mathrm{k}}$ represents the set of pixels belonging to the intermediate neighbourhood of pixel $\mathrm{x}_{\mathrm{k}}$ and $\mathrm{N}_{\mathrm{R}}$ is the cardinality of $\mathrm{N}_{\mathrm{k}}$. The influence of the second term of equation (13) on the labelling of each pixel is weighted by the parameter $n_{R}$ $\left(n_{R} \in\left[0, N_{R}\right]\right)$. When the image suffers from strong noise presence a high value for the parameter $n_{R}$ must be used and inversely, a low value for the parameter $n_{R}$ must be used when the signal to noise ratio (SNR) is large.

By substituting equation (2) to equation (13):

$$
J_{a b}=\sum_{i=1}^{c} \sum_{k=1}^{N} u_{i k}^{p} \cdot\left\|\frac{y_{k}-b_{k}}{a_{k}}-v_{i}\right\|^{2}+\frac{n_{R}}{N_{R}} \cdot \sum_{i=1}^{c} \sum_{k=1}^{N} u_{i k}^{p} \cdot\left(\sum_{x_{r} \in N_{k}}\left\|\frac{y_{r}-b_{r}}{a_{r}}-v_{i}\right\|^{2}\right)
$$

Thus, the problem is the minimization of the above objective function (equation (14)) with respect to $\mathrm{u}, \mathrm{v}, \mathrm{a}, \mathrm{b}$ :

$$
\min _{\left.\left.U,\left\{v_{i}\right\}_{i=1}^{C},\left\{a_{k}\right\},\right\}=1,\left\{b_{k}\right\},\right\}_{k=1}^{N}} J_{a b},
$$

subject to $U \in U$.

The objective function $\mathrm{J}_{\mathrm{ab}}$ can be minimized by taking the first derivatives of $\mathrm{J}_{\mathrm{ab}}$ with respect to $\mathrm{u}_{\mathrm{i}}, \mathrm{v}_{\mathrm{k}}, \mathrm{a}_{\mathrm{k}}, \mathrm{b}_{\mathrm{k}}$ and setting them to zero. This results in four necessary but not sufficient conditions for $J_{a b}$ to be in a local extrema.

The minimization of equation (14) could be solved using one Lagrange multiplier: 


$$
F_{a b}=\sum_{i=1}^{c} \sum_{k=1}^{N}\left(u_{i k}^{p} \cdot D_{i k}+\frac{n_{R}}{N_{R}} \cdot u_{i k}^{p} \cdot \gamma_{i}\right)+\lambda \cdot\left(1-\sum_{i=1}^{c} u_{i k}\right)
$$

where

$$
D_{i k}=\left\|\frac{y_{k}-b_{k}}{a_{k}}-v_{i}\right\|^{2} \text { and } \gamma_{i}=\sum_{y_{r} \in N_{k}}\left\|\frac{y_{r}-b_{r}}{a_{r}}-v_{i}\right\|^{2}
$$

Taking the derivative of $\mathrm{F}_{\mathrm{ab}}$ with respect to $\mathrm{u}_{\mathrm{ik}}$ and setting it to zero:

$$
\begin{aligned}
& \left.\frac{\partial F_{a b}}{\partial u_{i k}}\right|_{u_{i k}=u_{i k}^{*}}=\left.\left(p \cdot u_{i k}^{p-1} \cdot D_{i k}+\frac{n_{R} \cdot p}{N_{R}} \cdot u_{i k}^{p-1} \cdot \gamma_{i}-\lambda\right)\right|_{u_{i k}=u_{i k}^{*}}=0 \Rightarrow \\
& u_{i k}^{*}=\left(\frac{\lambda}{p \cdot\left(D_{i k}+\frac{n_{R}}{N_{R}} \cdot \gamma_{i}\right)}\right)^{1 /(p-1)} \\
& \sum_{j=1}^{c} u_{j k}=1 \forall k \Rightarrow \sum_{j=1}^{c}\left(\frac{\lambda}{p \cdot\left(D_{j k}+\frac{n_{R}}{N_{R}} \cdot \gamma_{j}\right)}\right)^{1 /(p-1)}=1 \\
& \Rightarrow \lambda=\frac{p}{\left(\sum_{j=1}^{c}\left(\frac{1}{\left(D_{j k}+\frac{n_{R}}{N_{R}} \cdot \gamma_{j}\right)}\right)^{1 /(p-1)}\right)^{p-1}}
\end{aligned}
$$

Substituting back to equation (19), we have:

$$
u_{i k}^{*}=\frac{1}{\sum_{j=1}^{c}\left(\frac{D_{i k}+\frac{n_{R}}{N_{R}} \cdot \gamma_{i}}{D_{j k}+\frac{n_{R}}{N_{R}} \cdot \gamma_{j}}\right)^{1 /(p-1)}}
$$

Taking the derivative with respect to $v_{i}$ it yields:

$$
\left(\left.\sum_{k=1}^{N}\left(u_{i k}^{p} \cdot\left(\frac{y_{k}-b_{k}}{a_{k}}-v_{i}\right)+\sum_{k=1}^{N} u_{i k}^{p} \cdot \frac{n_{R}}{N_{R}} \cdot \sum_{y_{r} \in N_{k}}\left(\frac{y_{r}-b_{r}}{a_{r}}-v_{i}\right)\right)\right|_{v_{i}=v_{i}^{*}}=0 \Rightarrow\right.
$$




$$
v_{i}^{*}=\frac{\sum_{k=1}^{N} u_{i k}^{p} \cdot\left(\left(\frac{y_{k}-b_{k}}{a_{k}}\right)+\frac{n_{R}}{N_{R}} \cdot \sum_{y_{r} \in N_{k}}\left(\frac{y_{r}-b_{r}}{a_{r}}\right)\right)}{\left(1+n_{R}\right) \cdot \sum_{k=1}^{N} u_{i k}^{p}} .
$$

Taking the derivative of $F_{a b}$ with respect to $a_{k}$ and setting to zero we have:

$$
\begin{gathered}
\left.\left(\sum_{i=1}^{c} \frac{\partial}{\partial a_{k}} \sum_{k=1}^{N} u_{i k}^{p} \cdot\left(\frac{y_{k}-b_{k}}{a_{k}}-v_{i}\right)^{2}\right)\right|_{a_{k}=a_{k}^{*}}=0 \Rightarrow \\
a_{k}^{*}=\left(y_{k}-b_{k}\right) \cdot \frac{\sum_{i=1}^{c} u_{i k}^{p}}{\sum_{i=1}^{c} u_{i k}^{p} \cdot v_{i}}
\end{gathered}
$$

Similarly, taking the derivative of $F_{a b}$ with respect to $b_{k}$ we have:

$$
b_{k}^{*}=y_{k}-a_{k} \frac{\sum_{i=1}^{c} u_{i k}^{p} \cdot v_{i}}{\sum_{i=1}^{c} u_{i k}^{p}}
$$

Thus, the algorithm for shading correction of the acquired infrared image and the estimation both of the shading-free image and the multiplicative and additive shading components can be summarized below:

1) Select randomly centroids for the two ( $c=2$ ) clusters (background, other) and set $a_{k}$ to values near to one and $b_{k}$ to values near to zero.

2) Update the partition matrix using equation (22).

3) Find the centroids of each cluster using equation (24).

4) Estimate the multiplicative shading component using equation (26).

5) Filter the multiplicative shading component using an iterative low pass filter.

6) Estimate the additive shading component using equation (27).

Repeat steps 2 to 6 until:

$$
\left\|v_{\text {new }}-v_{\text {old }}\right\|<\varepsilon
$$

where $\|\cdot\|$ is the Euclidean norm, $\mathrm{v}$ is the vector of cluster centers and $\varepsilon$ is a small real number.

A low pass filter is applied multiple times on the multiplicative shading component at step 5 according to the hypothesis that the multiplicative shading component is spatially smooth (neighbor pixels have similar intensity values). It prevents the method from enclosing to an endless loop of estimating the same values for the multiplicative and additive shading components or from converging to a local minimum. 
After the estimation of the multiplicative and additive shading components $\hat{A}$ and $\hat{B}$ from their corresponding vector forms $\alpha_{k}$ and $b_{k}$ the original shading-free image is obtained by applying the inverse of the image formation model (equation (2)).

\section{Experimental results}

The proposed algorithm is evaluated on both real acquired infrared images and artificial created images. The original images acquired using the experimental device already described. On the other hand the artificial images are created using the following procedure. Each artificial image created by the weighed sum of two artificial images. The first image is constructed using an artificial vessel-like network. This network consists of connected lines of different widths with junctions and bifurcations and multiple low pass filtering to simulate the blurriness of the edges which is apparent to the real images due to the blood flow and scattering effects. The second artificial image is used to simulate the non-uniform image background which created by applying an iterative spatial low pass Gaussian filter with a large region of support (window size) to a real infrared image. Figure 2 shows on the left two real infrared images acquired by devices such those described on the image acquisition section and on the right the corresponding estimations of the shading-free images after the application of the proposed method. Figure 3a shows a real ROI infrared image while Figure $3 b$ shows the corresponding estimation of the shading-free image after the application of the proposed method. Figure $4 \mathrm{a}$ and Figure $4 \mathrm{~b}$ show two artificial created images by the procedure described above while Fig. $4 \mathrm{c}$ and Fig. $4 \mathrm{~d}$ show the estimations of the shading-free images obtained after the estimation of the multiplicative and the additive shading component using the proposed method and inverting the liner model (equation (2)). As it can be observed from these figures, the proposed method has removed the majority of shading and has decreased the variations in illumination both in case of real and artificial infrared images and thus satisfies the goal of a retrospective shading correction method.

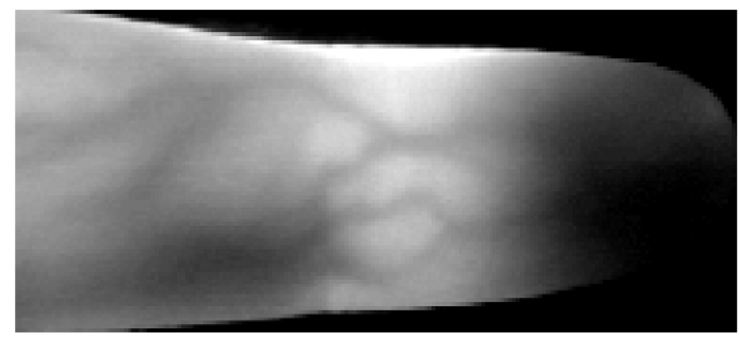

a.

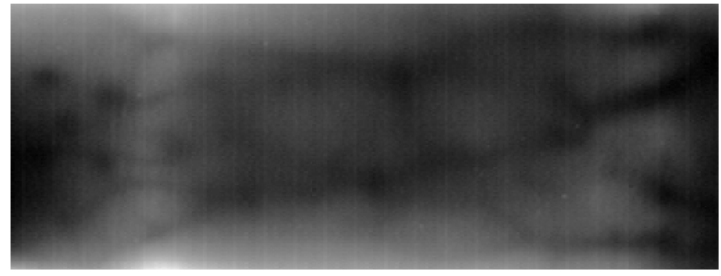

c.

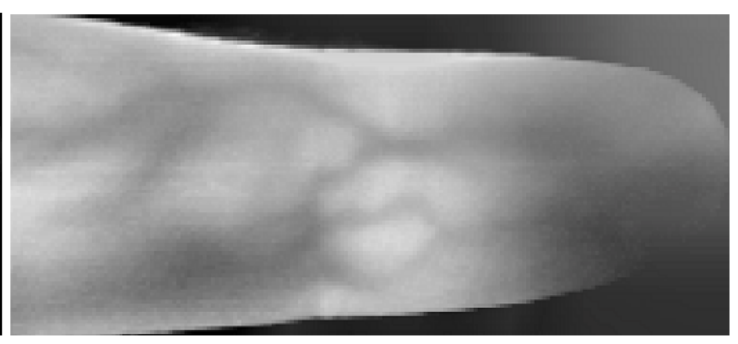

b.

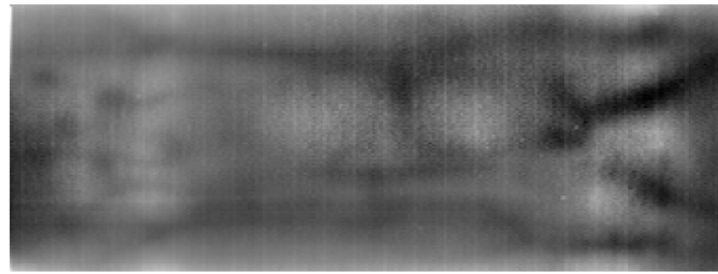

d.

Figure 2. a, c. Real infrared images (left) and b, d. the corresponding estimations of the shading-free images after the application of the proposed method 


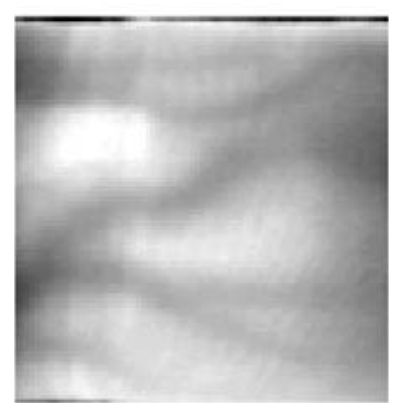

a.

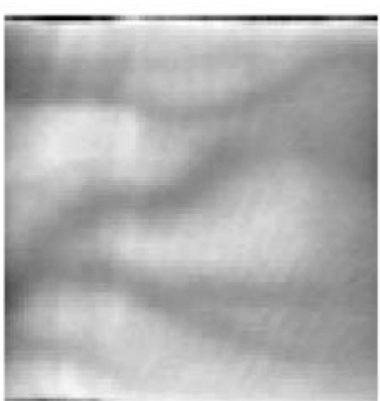

b.

Figure 3. a. ROI (Region Of Interest) extracted from a real infrared image of a finger and $\mathrm{b}$. the corresponding shadingfree image of a finger obtained after the application of the proposed method

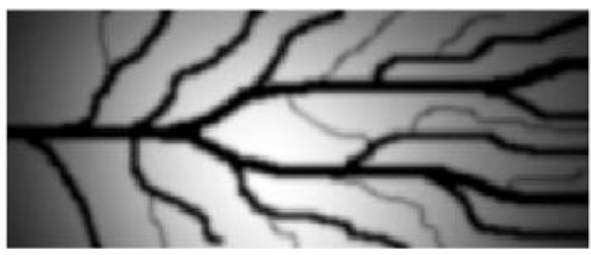

a.

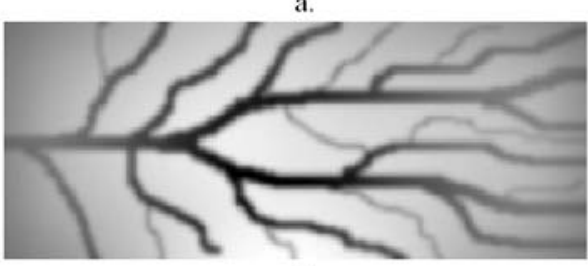

c.

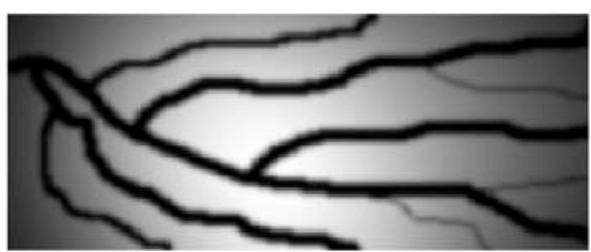

b.

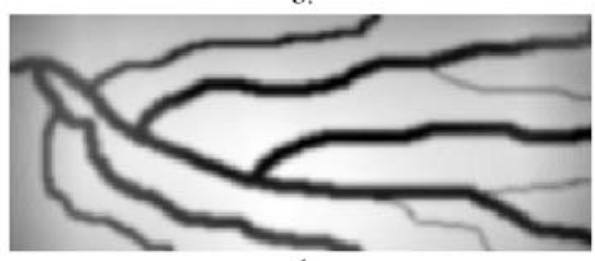

d.

Figure 4. a, b. Artificial infrared images of a finger and c, d. shading-free image of a finger obtained after the application of the proposed method

The quantitative evaluation of the proposed method was performed in our real finger database and in our artificial finger image database. The evaluation is performed, using the signal to noise ratio (or equivalently the reciprocal of the coefficient of variations) as a criterion of intensity homogeneity.

In general, the coefficient of variations (CV) is a normalized measure of dispersion of a probability distribution. It is defined as the ratio of the standard deviation $\sigma$ to the mean $\mu$ :

$$
C V=\frac{\sigma}{\mu}
$$

It is defined only for non-zero mean and it is most useful for variables that are always positive. It is also known as unitized risk.

Traditionally, SNR has been defined as the ratio of the average signal value to the standard deviation of the signal value. It is the reciprocal of the coefficient of variation:

$$
S N R=\frac{\mu}{\sigma}=\frac{1}{C V}
$$


In substance, the performance of the proposed method is expressed by the reduction/increase of intensity variations in the image after shading correction. Table 1 summarizes the results of the estimation of SNR for the images shown in figures 2-4 which are randomly selected both from real and artificial image databases.

Table 1. Signal to Noise Ratio before and after shading correction

\begin{tabular}{|c|c|c|c|}
\hline \multicolumn{4}{|c|}{ Real Image Database } \\
\hline \multicolumn{2}{|c|}{ Acquired Images } & \multicolumn{2}{|c|}{ Shading-free Images } \\
\hline Image 1 (Fig.2a) & 1.387 & Image 1 (Fig.2b) & 2.663 \\
\hline Image 2 (Fig.2c) & 1.585 & Image 2 (Fig.2d) & 2.861 \\
\hline Image 3 (Fig.3a) & 3.565 & Image 3 (Fig.3b) & 3.895 \\
\hline \multicolumn{4}{|c|}{ Artificial Image Database } \\
\hline \multicolumn{2}{|c|}{ Original Images } & \multicolumn{2}{|c|}{ Shading-free Images } \\
\hline Image 1 (Fig.4a) & 1.467 & Image 1 (Fig.4c) & 2.925 \\
\hline Image 2 (Fig.4b) & 1.447 & Image 2 (Fig.4d) & 2.393 \\
\hline
\end{tabular}

From the results presented in the above figures and table, it is obvious that the proposed method reduces the shading in the distorted images and delivers images with a larger degree of intensity homogeneity while retaining the larger amount of information content. The average improvement in the SNR is 33\% for the whole set of images belonging to real image database and $46 \%$ for the whole set of images belonging to artificial image database.

Unfortunately, there is no exist a publicly available infrared image database in order to compare the proposed method with those have been already presented in the literature. Also, the numerical results presented in these methods are for different image modalities i.e. MRI, TEM, Retinal images and there is no a base to perform a fair comparison. Moreover, in contrast to the proposed method which has a few parameters with predefined values, existing methods require parameter tuning. The majority of authors construct artificial images to evaluate their methods ${ }^{[16]}$, three sets of twenty differently structured artificial images were generated. Finally, both our real and artificial image databases have a few number of images (each has ten images) and they do not yet appropriate for releasing in the web. This comparison will be included in our future work.

\section{Discussion}

The main goal of a method such that proposed in this paper is to be used as a preprocessing step in automatic image processing and analysis algorithms such as image segmentation. Thus, a successful application of this method i.e, in an image segmentation task would be to remove the background inhomogeneities and to reveal information about veins that may be hidden due to shading or the presence of noise.

The first acquired image shown in figure 2 (see Figure 2a) suffers from shading and non-uniform illumination produced mainly due to the image acquisition system. The average luminosity is higher in the top part of the image than in the bottom which has a large dark region on the right and also a smaller dark region on the left. On the other hand, the luminosity of the top part of the image is higher and in the middle is saturated. Finally, the middle part of the image is brighter than both the left and right parts. All these variations in the luminosity produced mainly due to the relative location of the leds regarding the location of the finger in the image acquisition system and due to the variations of thickness of the finger. These variations, which are present in all parts of the acquired image may deteriorate the performance of an image segmentation method based on differences in pixel intensities, because some parts of the image, that have similar intensities with shading or background, may erroneously classified as veins. The application of the proposed method reduces the majority of these variations, reveals significant information about veins and delivers in the image segmentation method a more uniform image. 
The second acquired image also shown in figure 2 (see Figure 2c) suffers from shading and non-uniform illumination produced mainly due to the image acquisition system. In this case, due to the setup of the acquisition system the main part of the finger has been captured in the acquired image and thus there is no a dark background. Even in such case the image presents similar variations in intensity with the first image of figure 2 . The image is darker in the left and the right side and significant shading appears in the middle. The application of the proposed method reduces the majority of these variations, reveals significant information about veins (especially in the left side of the image) and delivers in the image segmentation method a more uniform image.

The case of the image shown in figure 3 is slightly different. Figure 3 presents a ROI image extracted from an acquired image by manually cropping the desired region. This part of image suffers mainly from saturation (large white blob in the upper left part of the ROI) and shading in the left and right side of the ROI. The application of the proposed method uniforms the intensity (large blob has the same levels of intensity with the other background parts) in the ROI image and reduces the shading in both left and right sides of the ROI. This image (see Figure 3a) is acceptable (visual inspection) for processing by image analysis algorithms and does not require preprocessing. The reason for which has been included in this paper is to illustrate that the proposed method satisfies the goal of the retrospective shading correction. Thus, it removes shading and inhomogeneities without distorting the shading free parts of the image.

The artificial images shown in figure 4, constructed in order to simulate the illumination conditions present in a real image acquisition system. Thus, shading appears on the left and the right part of the image and the average luminosity is higher in the middle part of the image. After the application of the proposed method the background is significantly more uniform than in the original image and the variations in the luminosity have been dramatically decreased. In all cases, it is worth to mention the degree of smoothness of the resulted images.

By observing the images in figures 2-4, one inference that can be easily exported is that the shading-free images produced after the application of the proposed method are more blurred compared with the acquired images. This is reasonable due to the application of the modified FCM algorithm on the background part of the image. The modified FCM algorithm in step 5 filters the multiplicative shading component using an iterative low pass filter. The result of this step is the removal of high frequencies from the multiplicative shading component which in turn affects the frequency content of the shading-free image. Although, this effect gives the sense that the shading-free image has lower quality than the original image does not significantly influence the performance of automatic image analysis algorithms that may be applied in the sequel. In image processing, visual inspection is not the safer method for comparison between images produced using different methods. Moreover, in some cases, this blurring effect produced by shading correction may be required, as a significant preprocessing step, such in the case of the segmentation of vasculature of infrared images by an automatic image segmentation method.

As the above results show, the proposed method performs well in the majority of cases and achieves the main goal of retrospective shading correction. It reduces the majority of shading while it does not introduce shading if the image is shading free. However, no scientific method is perfect. Every method has also drawbacks. Thus, the main drawback of the proposed method is the random selection of the prototypes of classes in the first step of the modified FCM algorithm. Although, the selection has been restricted, by the extraction of the pixels belongs with high confidence to background, this selection affects the performance of the overall method in terms of speed and computation complexity.

An additional interesting task would be the study of the variations in the performance of the proposed method due to the presence of noise in the acquired images. Different types and levels of noise can be used to artificially distort the acquired images and then the proposed method could be applied to remove the shading and the non-uniform background under these adverse conditions. Depending on the type of noise the behavior of the proposed method may be varied a lot. Thus, the study of the effect of noise will lead on the adaptation and/or the modification of the proposed method in order to robustly perform under extreme conditions. These conditions, although seems to be unrealistic may be produced by a low quality acquisition system and its non-careful setup. The above issues would be included in our future work. 


\section{Conclusions}

In this paper a novel brightness normalization method is proposed to eliminate the non-uniform illumination effects. The method is based on the application of a FCM algorithm only on the background part of the acquired image, where the objective function is modified to take into account local information of each pixel in the estimation of the multiplicative and the additive shading components. The modified FCM algorithm is iterative, as the standard FCM, and at each iteration the multiplicative and the additive shading components are re-estimated based on the cluster centers and the memberships of each pixel in a specific cluster. Brightness correction is performed by the inverse of the image formation model after FCM convergence.

Experiments were conducted in a database of both real and artificial infrared images. The experimental results show that the proposed method decreases significantly the non-uniform illumination effects and does not introduce brightness variations if the background is uniform.

\section{Acknowledgments}

The authors would like to thank the reviewers for their valuable comments, which improve the quality of this research paper.

\section{References}

[1] J.C. Russ, The image processing handbook, Second Edition, IEEE press, Boca Raton, 1995.

[2] I.T. Young, J.J. Gerbrands, and L.J. Vliet. Image Processing Fundamentals, CRC press, Boca Raton. 1998: 51.1-51.81.

[3] B. Likar, J.B.A. Maintz, M.A. Viergever, and F. Pernus. Retrospective shading correction by entropy minimization, Journal of Microscopy. 2000; 197(3): 285-295. PMid:10692132 http://dx.doi.org/10.1046/j.1365-2818.2000.00669.x

[4] S.E. Umbaugh, Computer vision and image processing, Prentice-Hall, New York, 1998. PMid:9604704

[5] R.M. Haralick, S.R. Sternberg and X. Zhuang. Image Analysis Using Mathematical Morphology. IEEE Trans. Pattern Anal. Machine Intell. 1987; 9: 532-550. PMid:21869411 http://dx.doi.org/10.1109/TPAMI.1987.4767941

[6] C. Munzenmayer, F. Naujokat, S. Muhldorfer, and T. Wittenberg, Enhancing Texture Analysis by Color Shading Correction, in 9. Workshop Farbbildverarbeitung, K.-H. Franke, ed., Zentrum für Bild- und Signalverarbeitunge.V., (Ilmenau), 2003.

[7] M. Foracchia, E. Grisan and A. Ruggeri. Luminosity and contrast normalization in retinal images. Medical Image Analysis. 2005: 179-190. PMid:15854840 http://dx.doi.org/10.1016/j.media.2004.07.001

[8] T. Tasdizen, E. Jurrus and R. Whitaker, Non-uniform illumination correction in transmission electron microscopy, Third MICCAI Workshop, 2008, New York.

[9] Reyes-Aldasoro, C.C., Retrospective shading correction algorithm based on signal envelope estimation, Electronics Letters. 23 April 2009; 45(9): 454-456.

[10] Young IT, Shading correction: compensation for illumination and sensor inhomogeneities, Curr Protoc Cytom. Chapter 2: Unit $2.11,2001$.

[11] M. Vlachos and E. Dermatas. Blind retrospective shading correction using a multi-objective minimization criterion. Computerized Medical Imaging and Graphics. 2012; 36(6): 501-513. PMid:22564545 http://dx.doi.org/10.1016/j.compmedimag.2012.04.002

[12] W. Chen, M.L. Giger. A fuzzy c-means (FCM) based algorithm for intensity inhomogeneity correction and segmentation of MR images. IEEE International Symposium on Biomedical Imaging: Nano to Macro. 2004; 2: 1307-1310.

[13] M.N. Ahmed, S.M. Yamany, N. Mohamed, A.A. Farag, T. Moriarty. A modified fuzzy c-means algorithm for bias field estimation and segmentation of MRI data. IEEE Transactions on Medical Imaging. 2002; 21: 193-199. PMid:11989844 http://dx.doi.org/10.1109/42.996338

[14] M.N. Ahmed, S.M. Yamany, N. Mohamed, A.A. Farag, T. Moriarty, Bias field estimation and adaptive segmentation of MRI data using modified fuzzy C-means algorithm, in Proc. IEEE Int. Conf. Computer Vision and Pattern Recogn. 1999; 1: $250-255$.

[15] M. Vlachos and E. Dermatas, Removing non uniform illumination effects in infrared images. Advanced Topics in Scattering Theory and Biomedical Engineering. 2010: 153-160.

[16] D. Tomazevic, B. Likar, F. Pernus. Comparative evaluation of retrospective shading correction methods. J Microsc. 2002; 212-223. PMid:12460452 http://dx.doi.org/10.1046/j.1365-2818.2002.01079.x 